\title{
The flow induced by a rotationally oscillating and translating circular cylinder
}

\author{
By S. C. R. DENNIS ${ }^{1}$, P. NGUYEN ${ }^{1}$ \\ AND SERPIL KOCABIYIK ${ }^{2}$ \\ ${ }^{1}$ Department of Applied Mathematics, University of Western Ontario, London, \\ Ontario, Canada N6A 5B7 \\ ${ }^{2}$ Department of Mathematics and Statistics, Memorial University of Newfoundland, \\ St. John's, Newfoundland, Canada A1C 5S7
}

(Received 28 January 1997 and in revised form 13 September 1999)

The temporal development of two-dimensional viscous incompressible flow induced by an impulsively started circular cylinder which performs time-dependent rotational oscillations about its axis and translates at right angles to this axis is investigated. The investigation is based on the solutions of the unsteady Navier-Stokes equations. A series expansion for small times is developed. The Navier-Stokes equations are also integrated by a spectral-finite difference method for moderate values of time for both moderate and high Reynolds numbers. The numerical method is checked with the results of the analytical solution. The effects of the Reynolds number and of the forcing Strouhal number $S$ on the laminar asymmetric flow structure in the near-wake region are studied. The lift and drag coefficients are also extracted from numerical results. An interesting phenomenon has been observed both in the flow patterns and in the behaviour of drag coefficients for $S=\pi / 2$ at Reynolds number $R=500$ and is discussed. For comparison purposes the start-up flow is determined numerically at a low Reynolds number and is found to be in good agreement with previous experimental predictions.

\section{Introduction}

The unsteady flow past an oscillating circular cylinder has long been of interest and has been reported in numerous works. The main interests in oscillating flows are due to the possibility of controlling and modifying the wake by means of mechanical excitations and the process of force generation due to vortex shedding. Many properties and applications of the oscillatory flows are reported in review articles by Berger \& Willie (1972), Bearman (1984) and Griffin \& Hall (1991) and also in a recent book by Sümer \& Fredsøe (1997).

Much of the prior work has dealt with the case of a steadily rotating (no forced oscillations) and translating circular cylinder (see for example Badr \& Dennis 1985; Badr et al. 1986, 1990; Coutanceau \& Menard 1985; Chang \& Chern 1991; Chen, Ou \& Pearlstein 1993; Nair, Sengupta \& Chauhan 1998). The work by Badr et al. (1990) is a joint theoretical and experimental study in the Reynolds number range $10^{3} \leqslant R \leqslant 10^{4}$ and for rotational to translational surface speed ratios between 0.5 and 3. Their theoretical results are based on numerical solutions of the two-dimensional Navier-Stokes equations for incompressible fluids. In their experimental study, the flow is generally two-dimensional except for the highest rotational rate of the cylinder, 
where the flow starts to develop three-dimensional and turbulent effects which appear at later times. In such cases, Badr et al. report that it is still possible to identify many common features of the experimental and calculated flows, particularly up to the time for which the flow continues to be laminar, and even beyond in some flow regions.

This present paper gives results for the laminar two-dimensional flow generated by an infinitely long circular cylinder. Initially the cylinder is at rest, and then suddenly starts to move with constant velocity at right angles to its axis and at the same time starts to perform time-dependent rotational oscillations about its axis. It is noted that purely two-dimensional shedding cannot be achieved in practice due to the end conditions. The three-dimensional transition of the near wake of a circular cylinder was investigated by Williamson (1988). In his experimental work three-dimensional structures in the wake are observed when $R>178$. Although physically the wake is three-dimensional, we believe, as is supported by the agreement between calculated results and experimental data, that it can be reasonably well represented by the present two-dimensional model.

From the standpoint of controlling laminar two-dimensional vortex shedding from a circular cylinder by using 'active control', the nature of the vortex shedding process can be significantly altered by cylinder rotation. Generally speaking, the control of flow physics and near-wake structure of a bluff body may take the form of global control, where the entire body is subjected to prescribed motion, or local control, involving localized application of unsteady blowing/suction or heating at specified positions on the surface of the stationary body. Attention here is focused on the case of global control where the time-dependent rotational oscillatory velocity of a circular cylinder is represented by $\alpha^{*}(\tau)=\alpha_{0} \sin (\omega \tau)$. Here $\alpha_{0}$ is the maximum angular velocity about the cylinder axis and $\omega=2 \pi F$ is the angular frequency of oscillation, where $F$ is the frequency of oscillation.

For a translating circular cylinder under rotational oscillations the flow field depends mainly on three dimensionless parameters. The first is the Reynolds number, defined as $R=2 a U / v$, where $U$ is the constant speed of translation of the cylinder, $a$ is the radius of the cylinder and $v$ is the coefficient of kinematic viscosity of the fluid. The second is the forcing Strouhal number $S$, defined as $S=a \omega / U$ (i.e. $S=2 \pi a F / U$ or $S=2 \pi f$ ) which characterizes the forced oscillation frequency. The third is the dimensionless peak rotation rate $\alpha_{m}=\alpha_{0} / U$. It is noted that the dimensionless time $t$ is related to the dimensional time $\tau$ through $t=U \tau / a$ and the dimensionless oscillatory velocity can be expressed in terms of dimensionless parameters by the relation $\alpha(t)=\alpha_{m} \sin (S t)$ or $\alpha(t)=\alpha_{m} \sin (2 \pi f t)$.

If a fluid is in relative motion past a bluff cylinder which is forced to vibrate over a range of frequencies near the Kármán vortex-shedding frequency, then resonant flowinduced oscillations of the cylinder occur, i.e. there is a lock-on or synchronization phenomenon, $f / f_{0} \sim 1$, where $f_{0}$ is the Kármán vortex-shedding frequency for the flow past the cylinder without oscillation. In the present study $f_{0}$ is normalized with the constant speed of the cylinder translation $U$ and the cylinder radius $a$; it varies with $R$ and remains practically constant (namely, at the value of 0.1 ) for $R \geqslant 300$. It may be noted that $f / f_{0}=S /(0.2 \pi)$. In a recent numerical work by Steggel \& Rockliff (1997), a hybrid discrete vortex method was used to discuss the effects of body shape on the lock-on characteristics in oscillatory flows over rectangular-sectioned cylinders. For unsteady flow two lock-on regimes were identified, with symmetric shedding occurring for longer rectangles and sufficiently high oscillation amplitudes and frequencies.

Relatively few studies have been carried out on problems concerning the effect 
The flow induced by a rotationally oscillating and translating circular cylinder 125

of time-dependent rotational oscillations on vortex-shedding processes and on the hydrodynamic forces. Experimental work of Taneda (1978) for $30 \leqslant R \leqslant 300$ demonstrates how the wake of the two-dimensional steady flow past a circular cylinder can be substantially modified by high values of the control parameters. In fact, the flow visualizations indicated that for the cases of $R=40$ and $23 \pi<S<54 \pi$ at very high peak rotation rates, the vortex-shedding process could be nearly eliminated. A lock-on state was observed in the study by Okajima, Takata \& Asanuma (1975) when the applied frequency is that of natural vortex shedding. They examined the forces acting on a rotationally oscillating cylinder when $40 \leqslant R \leqslant 6 \times 10^{3}, 0.2 \leqslant \alpha_{m} \leqslant 1.0$ and $0.05 \pi \leqslant S \leqslant 0.3 \pi$. Investigations at comparable values of the control parameters $\alpha_{m}$ and $S$ at $R=300$ were also performed by Wu, Mo \& Vakili (1989). They found that the control parameters strongly affect the fluid forces acting on the cylinder. Vortex lock-on and control of the near-wake flow were also investigated by Tokumaru \& Dimotakis (1991) and Filler, Marston \& Mih (1991). An important finding by Tokumaru \& Dimotakis is that very large peak rotation rates can produce significant reduction in drag on the cylinder. Their experiments were conducted at the Reynolds number of $R=1.5 \times 10^{4}$. Filler et al. investigated the stability of the separated shear layers for small values of $\alpha_{m}$ when $250 \leqslant R \leqslant 1200$.

To our knowledge, from a survey of existing literature, only three purely numerical studies have been made on this problem: Lu \& Sato (1996), Chou (1997) and Baek \& Sung (1998). Finite-difference simulations were carried out using primitive variables except for the work of Chou. These studies have a common focus on when and how the vortex shedding is synchronized with the cylinder oscillation. A fractional-step method (see for example Kim \& Moin 1985) was utilized by Lu \& Sato and Baek \& Sung to analyse the vortex formation modes behind a cylinder. In the first, simulations were made in a range $0.1 \leqslant \alpha_{m} \leqslant 3.0$ and $\pi \leqslant S \leqslant 8.0 \pi$ at three values of the Reynolds number $R=200,10^{3}$, and $3 \times 10^{3}$ whereas in the second, numerical calculations were carried out over the range $0.11 \pi \leqslant S \leqslant 0.22 \pi$ and $0.09<\alpha_{m}<0.72$ at the fixed Reynolds number $R=110$; the effects of $\alpha_{m}$ and $S$ on the vortex formation were studied, and some basic patterns of vortex shedding were identified. According to the computed results of $\mathrm{Lu} \&$ Sato, the large-scale vortex structures in the near wake remain nearly the same for $R=200,10^{3}$ and $3 \times 10^{3}$ and it is found that the variation of the fluid forces is closely related to the evolution of the vortex formation in the near wake. Chou simulated the synchronization phenomena via an explicit time-marching algorithm for the governing equations. In his work computed results were reported for $200 \leqslant R \leqslant 10^{3}$ and $S \sim 2 \pi$ and $4 \pi$ at moderate values of $\alpha_{m}$.

In the present paper the two-dimensional flow caused by an infinitely long circular cylinder which is set in motion impulsively and translates with uniform velocity and also undergoes rotational oscillation is analysed by means of an accurate mathematical formulation. The instantaneous translation and rotation start at the same moment and the development with time of the flow can be studied in a coordinate frame which translates with the cylinder but does not rotate. Equations for the flow in terms of the stream function and vorticity in boundary-layer coordinates are presented. A perturbation series solution for small times is developed. The flow for longer times is computed numerically using the same spectral-finite difference method as used by Badr \& Dennis (1985) for integrating the unsteady Navier-Stokes equations and the grid size and time steps used have been carefully tested. The numerical method is checked for small times by comparison with the results of the analytical solution.

Numerical calculations are performed for moderate values of the time in the cases $R=500: S=\pi / 6, \pi / 5, \pi / 4, \pi / 3,2 \pi / 5, \pi / 2$ or $f / f_{0}=0.833,1.0,1.25,1.66,2.0,2.5$, and 
$R=10^{3}: S=\pi / 4, \pi / 2$ or $f / f_{0}=1.25,2.5$. The peak rotational rate $\alpha_{m}$ for these cases is taken to be 1 . The initial flow is also determined numerically for high values of the control parameters $\alpha_{m}=5 \pi^{2}$ and $S=20 \pi$ at $R=20$ and good qualitative agreement with the experimental findings of Taneda (1978) is found. For comparison purposes the flow around a steadily rotating and translating circular cylinder is calculated for the constant rotation rate $\alpha(t)=\alpha=3.5$ at $R=200$ and tested against existing numerical and experimental results. Good agreement with these results is found.

The most recent numerical studies of the flow induced by the torsional oscillations of an elliptic cylinder were made by Riley \& Wybrow (1995) and D'Alessio, Dennis \& Nguyen (1999). In the first, the case of a rotationally oscillating but not translating elliptic cylinder was considered whereas in the second the flow induced by a rotationally oscillating and translating elliptic cylinder was studied. It is noted that the flow structures in these cases are different from the present case and that some unusual and unexpected features in the wake such as the production of two co-rotating vortices, depending upon the forcing Strouhal number and the Reynolds number, occur in the present case. These are not seen in the cases of flow past an elliptic cylinder but are present in the accelerated circular cylinder case (see Badr, Dennis \& Kocabiyik 1996).

\section{Governing equations and method of solution}

At time $t=0$ the cylinder, whose axis coincides with the $z$-axis, starts to move with constant speed $U$ in the negative $x$-direction and at the same instant starts to rotate about the $z$-axis in the counterclockwise direction with the angular velocity $\alpha^{*}(\tau)$. A frame of reference is used in which the axes translate with the cylinder but are fixed in direction. Polar coordinates $(r, \theta)$ are taken with the origin at the centre of the cylinder. The unsteady Navier-Stokes equations in terms of the variables $(\xi, \theta)$, where $\xi=\ln (r / a)$, are given by Badr \& Dennis (1985) in the form

$$
\begin{gathered}
\mathrm{e}^{2 \xi} \frac{\partial \zeta}{\partial t}=\frac{2}{R}\left(\frac{\partial^{2} \zeta}{\partial \xi^{2}}+\frac{\partial^{2} \zeta}{\partial \theta^{2}}\right)-\frac{\partial \psi}{\partial \theta} \frac{\partial \zeta}{\partial \xi}+\frac{\partial \psi}{\partial \xi} \frac{\partial \zeta}{\partial \theta}, \\
\frac{\partial^{2} \psi}{\partial \xi^{2}}+\frac{\partial^{2} \psi}{\partial \theta^{2}}=\mathrm{e}^{2 \xi} \zeta .
\end{gathered}
$$

Here $\psi$ is the stream function and $\zeta$ is the (negative) scalar vorticity. These quantities are all dimensionless as defined in Badr \& Dennis (1985). The boundary conditions necessary to obtain a solution in the region $\xi \geqslant 0,0 \leqslant \theta \leqslant 2 \pi$ are

$$
\begin{gathered}
\psi=0, \quad \frac{\partial \psi}{\partial \xi}=-\alpha(t) \quad \text { at } \xi=0, \\
\mathrm{e}^{-\xi} \frac{\partial \psi}{\partial \xi} \rightarrow \sin \theta, \quad \mathrm{e}^{-\xi} \frac{\partial \psi}{\partial \theta} \rightarrow \cos \theta \quad \text { as } \xi \rightarrow \infty, \\
\zeta \rightarrow 0 \quad \text { as } \xi \rightarrow \infty, \\
\psi(\xi, \theta, t)=\psi(\xi, \theta+2 \pi, t), \quad \zeta(\xi, \theta, t)=\zeta(\xi, \theta+2 \pi, t) .
\end{gathered}
$$

The dimensional rotational velocity $\alpha^{*}(\tau)$ is made dimensionless through $\alpha^{*}(t)=$ $(U / a) \alpha(t)$. It is noted that care is necessary to satisfy properly the conditions (2.3) at large distances; this can be achieved by employing equivalent integral conditions on the vorticity (see Dennis \& Quartapelle 1989 and Dennis \& Kocabiyik 1991). 
The flow induced by a rotationally oscillating and translating circular cylinder 127

Badr \& Dennis (1985) have developed a method of solution of (2.1) and (2.2) by means of the substitutions

$$
\begin{aligned}
& \psi(\xi, \theta, t)=\frac{1}{2} F_{0}(\xi, t)+\sum_{n=1}^{\infty} F_{n}(\xi, t) \cos n \theta+f_{n}(\xi, t) \sin n \theta, \\
& \zeta(\xi, \theta, t)=\frac{1}{2} G_{0}(\xi, t)+\sum_{n=1}^{\infty} G_{n}(\xi, t) \cos n \theta+g_{n}(\xi, t) \sin n \theta .
\end{aligned}
$$

The equations governing the functions in (2.4) are

$$
\frac{\partial^{2} F_{0}}{\partial \xi^{2}}=\mathrm{e}^{2 \xi} G_{0}, \quad \frac{\partial^{2} F_{n}}{\partial \xi^{2}}-n^{2} F_{n}=\mathrm{e}^{2 \xi} G_{n}, \quad \frac{\partial^{2} f_{n}}{\partial \xi^{2}}-n^{2} f_{n}=\mathrm{e}^{2 \xi} g_{n},
$$

and

$$
\begin{aligned}
\mathrm{e}^{2 \xi} \frac{\partial G_{0}}{\partial t} & =\frac{2}{R} \frac{\partial^{2} G_{0}}{\partial \xi^{2}}+\sum_{m=1}^{\infty} \frac{\partial}{\partial \xi}\left[m\left(F_{m} g_{m}-f_{m} G_{m}\right)\right], \\
\mathrm{e}^{2 \xi} \frac{\partial G_{n}}{\partial t} & =\frac{2}{R}\left(\frac{\partial^{2} G_{n}}{\partial \xi^{2}}-n^{2} G_{n}\right)-\frac{1}{2} n f_{n} \frac{\partial G_{0}}{\partial \xi}+\frac{1}{2} S_{n}, \\
\mathrm{e}^{2 \xi} \frac{\partial g_{n}}{\partial \tau} & =\frac{2}{R}\left(\frac{\partial^{2} g_{n}}{\partial \xi^{2}}-n^{2} g_{n}\right)+\frac{1}{2} n F_{n} \frac{\partial G_{0}}{\partial \xi}+\frac{1}{2} T_{n} .
\end{aligned}
$$

Here $S_{n}$ and $T_{n}$ are nonlinear sums

$$
\begin{aligned}
S_{n}=\frac{\partial F_{0}}{\partial \xi} n g_{n} & +\sum_{m=1}^{\infty}\left[\left((m-n) F_{j}+k F_{k}\right) \frac{\partial g_{m}}{\partial \xi}-\left(j f_{j}+k f_{k}\right) \frac{\partial G_{m}}{\partial \xi}\right. \\
+ & \left.m\left(\frac{\partial F_{j}}{\partial \xi}+\frac{\partial F_{k}}{\partial \xi}\right) g_{m}-m\left(\operatorname{sgn}(m-n) \frac{\partial f_{j}}{\partial \xi}+\frac{\partial f_{k}}{\partial \xi}\right) G_{m}\right], \\
T_{n}=-\frac{\partial F_{0}}{\partial \xi} n G_{n} & +\sum_{m=1}^{\infty}\left[\left(k f_{k}-j f_{j}\right) \frac{\partial g_{m}}{\partial \xi}-\left((m-n) F_{j}-k F_{k}\right) \frac{\partial G_{m}}{\partial \xi}\right. \\
& \left.-m\left(\frac{\partial F_{j}}{\partial \xi}-\frac{\partial F_{k}}{\partial \xi}\right) G_{m}-m\left(\operatorname{sgn}(m-n) \frac{\partial f_{j}}{\partial \xi}-\frac{\partial f_{k}}{\partial \xi}\right) g_{m}\right],
\end{aligned}
$$

where $j=|m-n|, k=m+n$ and $\operatorname{sgn}(m-n)$ denotes the sign of $(m-n)$, with $\operatorname{sgn}(0)=0$. The boundary conditions necessary to solve the equations (2.5) and (2.6) are, when $\xi=0$

$$
\begin{aligned}
& F_{0}=0, \quad \frac{\partial F_{0}}{\partial \xi}=-2 \alpha(t), \\
& F_{n}=f_{n}=\frac{\partial F_{n}}{\partial \xi}=\frac{\partial f_{n}}{\partial \xi}=0,
\end{aligned}
$$

and as $\xi \rightarrow \infty$

$$
\begin{gathered}
\mathrm{e}^{-\xi} F_{0} \rightarrow 0, \quad \mathrm{e}^{-\xi} \frac{\partial F_{0}}{\partial \xi} \rightarrow 0, \quad G_{0} \rightarrow 0, \\
\mathrm{e}^{-\xi} F_{n}, \quad \mathrm{e}^{-\xi} \frac{\partial F_{n}}{\partial \xi} \rightarrow 0, \quad G_{n} \rightarrow 0 ; \quad \mathrm{e}^{-\xi} f_{n}, \quad \mathrm{e}^{-\xi} \frac{\partial f_{n}}{\partial \xi} \rightarrow \delta_{n, 1}, \quad g_{n} \rightarrow 0 .
\end{gathered}
$$


The conditions (2.8) express the fact that the velocity components of the flow at large distances relative to the motion of the cylinder reduce to those of a uniform stream parallel to $\theta=0$. The symbol $\delta_{n, 1}$ is the Kronecker delta.

Finally, the boundary conditions on the functions $F_{n}(\xi, t)$ and $f_{n}(\xi, t)$ are utilized to deduce sets of global conditions, termed integral conditions, on $G_{n}(\xi, t)$ and $g_{n}(\xi, t)$. These conditions,

$$
\begin{gathered}
\int_{0}^{\infty} \mathrm{e}^{2 \xi} G_{0}(\xi, t) \mathrm{d} \xi=2 \alpha(t), \quad \int_{0}^{\infty} \mathrm{e}^{(2-n) \xi} G_{n}(\xi, t) \mathrm{d} \xi=0, \\
\int_{0}^{\infty} \mathrm{e}^{(2-n) \xi} g_{n}(\xi, t) \mathrm{d} \xi=2 \delta_{n, 1},
\end{gathered}
$$

are employed in the solution procedure to ensure that all necessary conditions of the problem are satisfied. The use of integral conditions can be found in the works of Collins \& Dennis (1973), Badr et al. (1995a,b, 1996), Kocabiyik (1996a,b), Kocabiyik $\&$ Nguyen (1996), to mention a few of the various applications.

Equations (2.5) and (2.6) determine the development of the flow at some time after the impulsive start, but in the initial stages of the motion the boundary-layer coordinate $z$ can be introduced by the transformation

$$
\xi=k z, \quad k=2(2 t / R)^{1 / 2} .
$$

This is employed to transform all the appropriate equations together with the scalings of variables

$$
F_{n}=k F_{n}^{*}, \quad G_{n}=\frac{G_{n}^{*}}{k}, \quad f_{n}=k f_{n}^{*}, \quad g_{n}=\frac{g_{n}^{*}}{k},
$$

which maps the initial flow onto the scale of the boundary-layer thickness. This change of variables removes the singularity in the vorticity at $t=0$ due to the impulsive start. We emphasize that although boundary-layer coordinates are utilized, the full NavierStokes equations are to be solved and not the simplified boundary-layer equations. The governing equations and the boundary and integral conditions (2.5)-(2.9) are first transformed by means of (2.10) and (2.11) and the initial solution at $t=0$ is obtained following the work by Badr \& Dennis (1985). This initial solution is given by

$$
\begin{gathered}
\zeta^{*}(z, \theta, 0)=\frac{2}{\sqrt{\pi}}\left(a_{0}+2 \sin \theta\right) \mathrm{e}^{-z^{2}}, \\
\psi^{*}(z, \theta, 0)=-a_{0}\left[z(1-\operatorname{erf} z)+\frac{1}{\pi}\left(1-\mathrm{e}^{-z^{2}}\right)\right]+2\left[z \operatorname{erf} z-\frac{1}{\sqrt{\pi}}\left(1-\mathrm{e}^{-z^{2}}\right)\right] \sin \theta,
\end{gathered}
$$

where $a_{0}$ is a constant, defined by $a_{0}=\alpha(0)$.

From the initial expressions (2.12) and (2.13) we may now build up a perturbation solution in powers of $t$ following Badr \& Dennis (1985). The stream function and vorticity are expanded by using double power series in $k$ and $t$ :

$$
\psi^{*}(z, \theta, t)=\sum_{m=0}^{\infty} \sum_{n=0}^{\infty} \psi_{m n}^{*}(z, \theta) k^{m} t^{n}, \quad \zeta^{*}(z, \theta, t)=\sum_{m=0}^{\infty} \sum_{n=0}^{\infty} \zeta_{m n}^{*}(z, \theta) k^{m} t^{n} .
$$

The rotational velocity $\alpha(t)$ is also expanded in the form

$$
\alpha(t)=\sum_{n=0}^{\infty} a_{n} t^{n}
$$


The flow induced by a rotationally oscillating and translating circular cylinder 129

The process of derivation of $\psi_{m n}^{*}(z, \theta), \zeta_{m n}^{*}(z, \theta)$ follows very closely the procedures described by Badr \& Dennis (1985) and the differential equations and boundary conditions satisfied by them are easily found. Each of them can be expressed as a finite set of Fourier components in the coordinate $\theta$ with coefficients which are functions of the variable $z$. The series (2.14) are thus expressed in periodic terms in $\theta$ with coefficients depending on $z, t$ and $k$. On expansion in powers of $t$ and $k$ and equating to zero each coefficient of $k^{m} t^{n}$, we get the conditions which the Fourier components must satisfy. As a result of determining four composite functions $\zeta_{m n}^{*}(z, \theta)$ in the series (2.14), we obtain an expression for the vorticity of the form

$$
\zeta^{*}(z, \theta, t) \sim \zeta_{00}^{*}+t \zeta_{01}^{*}+k\left(\zeta_{10}^{*}+O(t)\right)+k^{2}\left(\zeta_{20}^{*}+O(t)\right),
$$

which is valid for small $t$ and large $R$. For our present purpose we shall only give the expression obtained for the surface vorticity. The expression (2.16) obtained by analytical means gives sufficient information to check the numerical solutions which are obtained by numerical integration of (2.5) and (2.6) subject to the conditions (2.7) and (2.9). In particular, we find for the surface vorticity

$$
\begin{aligned}
\zeta^{*}(0, \theta, t) \sim\left(4 \pi^{-1 / 2}+k-\frac{1}{4} \pi^{-1 / 2} k^{2}\right) \sin \theta+4 \pi^{-1 / 2} t & {\left[\left(a_{1}-\frac{4}{3} \pi^{-1} a_{0} \cos \theta\right)\right.} \\
+ & \left.\left(1+\frac{4}{3} \pi^{-1}\right) \sin 2 \theta\right] .
\end{aligned}
$$

In theory it is possible to determine analytical solutions for each $\zeta_{m n}^{*}$ and $\psi_{m n}^{*}$ satisfying the required boundary conditions. Exact solutions can be obtained for a few functions but they rapidly increase in complexity when $n>2$ for each $m$ and coefficients of the higher-order terms must be found by numerical methods. In the present work terms up to and including $t^{4}, k t^{3}, k^{2} t^{2}$ and $k^{3} t$ in $(2.14 a, b)$ are considered. Although this approach has been used by several authors, some of which include Collins \& Dennis (1973), Badr \& Dennis (1985), Badr et al. (1995a, 1996), Kocabiyik (1996a,b), there was no attempt to determine the range of convergence of the series (2.14). We now proceed to clarify this point. Since each solution $\zeta_{m n}^{*}$ is continuous and vanishes at infinity, $\zeta_{m n}^{*}$ must be bounded in the entire flow region $0 \leqslant z<\infty, 0 \leqslant \theta \leqslant 2 \pi$. It can be shown that $\zeta^{*}(z, \theta, t)$ is uniformly bounded by $M /[(1-t)(1-k)]$ for $0 \leqslant t<1$ and $0 \leqslant k<1$ where $M=\max \left\{\zeta_{m n}^{*}: m, n=0,1,2, \ldots\right\}$. A similar argument holds also for $\psi^{*}(z, \theta, t)$. Thus the power series expansions $(2.14 a, b)$ are valid in the range $0 \leqslant t<1$ and $0 \leqslant k$. It may be noted that $k=2(2 t / R)^{1 / 2}$ gives the greatest lower bound of $R$ as 8 when $k<1$ for $0 \leqslant t<1$.

\section{Numerical integration procedure}

In order to calculate the flow for any Reynolds number and large enough time, a numerical method is used which is similar to that given by Badr \& Dennis (1985). The solution is started in the boundary-layer variables by integrating (2.5) and (2.6) using (2.13) and (2.14) as initial conditions and (2.7) and (2.9) as boundary conditions. The use of the initial solutions (2.12) and (2.13) is essential for obtaining accurate results at small time. The use of the potential flow solution as an initial condition at $t=0$ has been frequently adopted by many researchers; however this will lead to inaccurate results following the start of the fluid motion. The effect of such inaccuracy on the large-time results is not known. An implicit method of Crank-Nicolson type is used to integrate the governing equations in time following the work by Badr \& Dennis (1985) and a given approximation is obtained by truncating the series $(2.4 a, b)$. In the present calculations the maximum number of terms used in the series $(2.4 a, b)$ is 
130

(a)

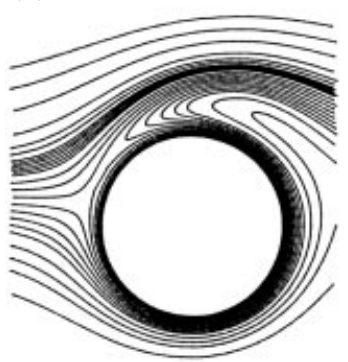

S. C. R. Dennis, P. Nguyen and S. Kocabiyik

(b)

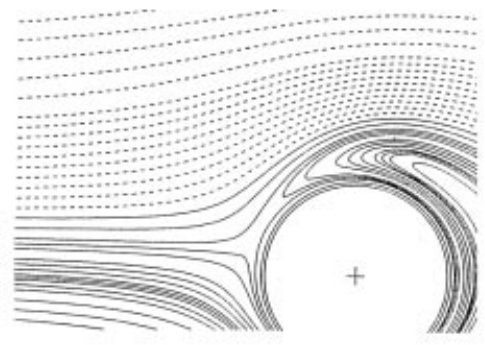

(c)

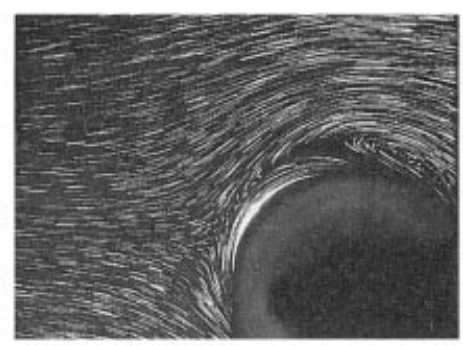

FIGURE 1. Comparison of computed ((a) present, (b) Chen et al. 1993) and experimental ((c) Coutanceau \& Menard (1985)) instantaneous streamlines in the case of steadily rotating and translating cylinder for $R=200, \alpha=3.25$ when $t=2.0$.

$N=30$. Checks were made for each $R$ at several typical values of $t$ to ensure that $N$ was large enough. This was done by increasing $N$ and observing that the solution did not change appreciably. A similar check was made by Badr et al. (1995b, p. 226) and the results found in the present case were essentially very similar and need not be given. The solution procedure requires knowledge of the surface vorticity distribution. The integral conditions (2.9) are used to calculate the values of functions $G_{n}$ and $g_{n}$ on the cylinder surface $(\xi=0)$ from which the required surface vorticity distribution can be obtained at every time step. In the work of Badr \& Dennis (1985) this was done by writing the integral as a quadrature formula which gives slow convergence at each time step. In the present study the surface vorticity was evaluated using the procedure given by Collins \& Dennis (1973, pp. 111-112) which is similar to the influence matrix technique in one-dimensional problems (see for example Daube 1992).

Because of the impulsive start, small time steps are needed to get past the singularity at $t=0$. For the cases of finite $R$ considered the integrations were all started by taking 10 time steps $\Delta t=10^{-4}$. The time step was then increased to $\Delta t=10^{-3}$ for the next 10 steps and then to $\Delta t=10^{-2}$ for the next 10 . Finally $\Delta t=0.025$ was taken for the rest of the solution. The grid size in the $z$-direction was taken as $\Delta z=0.05$ and the maximum value of $z$ was $z_{M}=8$. The values of grid sizes were to some extent chosen to be comparable with those used by Badr \& Dennis (1985), since these were found to be satisfactory and were checked carefully. A few comparable checks on different grids were made at one or two values of $t$ during the present calculations. The solutions obtained by fully numerical means are compared with the results obtained using expansions in powers of $t$ and good agreement is found between the results. Moreover, this scheme was tested against the documented steadily rotating and translating case; the tests indicate that the solutions are quite accurate.

\section{Results and discussion}

Before presenting the numerical results we first perform some accuracy checks on our numerical scheme. To do this, computations were carried out for the steadily rotating and translating case $(\alpha(t)=\alpha)$ when $R=200, \alpha=3.5$. The parameter values chosen allow comparison with the experimental results of Coutanceau \& Menard (1985). The time development of the flow for this case at $t=2$ is displayed in figure 1 and excellent agreement of our computed streamlines with previous experimental (Coutanceau \& Menard 1985) and numerical (Chen et al. 1993) results is obtained. Moreover, our numerical procedure at small times has been tested against the results 
The flow induced by a rotationally oscillating and translating circular cylinder 131

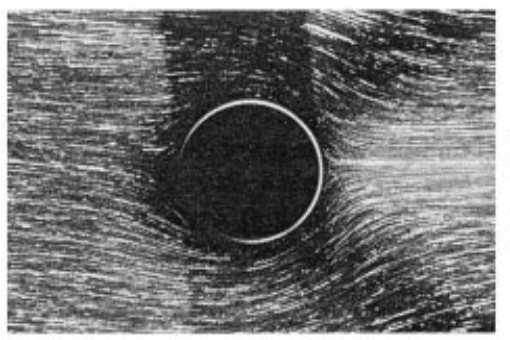

(a)
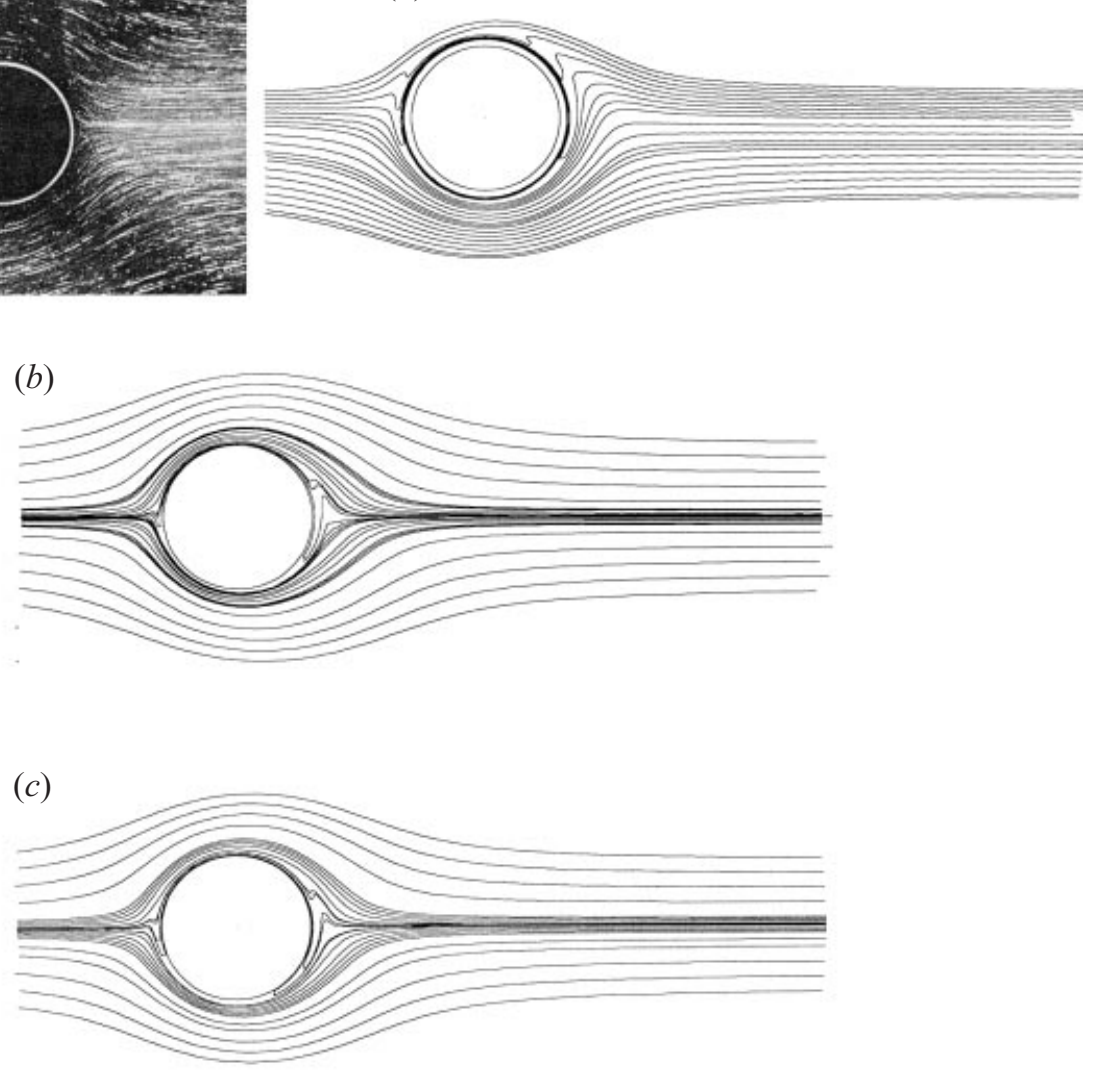

FiguRE 2. (a) Comparison of instantaneous streamlines with Taneda's (1978) flow visualizations for $R=20, S=20 \pi$ and $\alpha_{m}=5 \pi^{2}$ when $t=0.5$. $(b, c)$ Time-averaged flow field over one period of oscillation for $R=20, S=20 \pi$ and $\alpha_{m}=5 \pi^{2}$ at $(b) t=1.0$ and $(c) t=1.5$.

of the analytical solution; these tests indicate that the numerical results are quite accurate for small values of $t$. The drag and lift coefficients are computed using the formulae

$$
\begin{gathered}
C_{D}=\frac{2}{R} \int_{0}^{2 \pi}\left(\zeta-\frac{\partial \zeta}{\partial \xi}\right)_{\xi=0} \sin \theta \mathrm{d} \theta, \\
C_{L}=-\frac{2}{R} \int_{0}^{2 \pi}\left(\zeta-\frac{\partial \zeta}{\partial \xi}\right)_{\xi=0} \cos \theta \mathrm{d} \theta .
\end{gathered}
$$

We point out that at $t=0$ both $C_{D}$ and $C_{L}$ are infinite in magnitude due to the fact that the cylinder experiences infinite acceleration at that time and then decreases rapidly. For this reason $C_{D}$ and $C_{L}$ are plotted for $t>0$.

The flow around a circular cylinder performing rotational oscillations about its axis in a uniform flow was investigated by Taneda (1978) at Reynolds numbers in the range $30 \leqslant R \leqslant 300$ and forcing Strouhal numbers between 0 and $55 \pi$. He showed that the vortex shedding process as well as the reversed flow region behind the cylinder could be nearly eliminated at very high oscillation frequencies. The streamline pattern for the start-up flow is plotted in the case of $R=20$ when $S=20 \pi$ and $\alpha_{m}=5 \pi^{2}$ when $t=0.5$ and compared with the streamlines patterns of Taneda's experimental 
work at comparable values of $R$ and control parameters in figure 2(a). This case is rather like the potential flow case because no vortex sheds away from the cylinder and the flow pattern indeed resembles that of the potential flow except in the very small region near the cylinder. In figure $2(b, c)$ the calculated time-averaged flow field over one period of oscillation is plotted in the case of $R=20, S=20 \pi$ and $\alpha_{m}=5 \pi^{2}$ starting at $t=1.0$ and $t=1.5$, respectively. In these figures the flow is time-averaged over a complete period of oscillation of the cylinder up to the time at which it returns to the same position. These figures clearly indicate that flow separation and vortex shedding appear to have been completely inhibited for this high forcing Strouhal number range as predicted by Taneda and it may be noted that the calculated mean flows in figure $2(b, c)$ are almost steady state.

Numerical calculations are also given at $R=500$ and $R=10^{3}$. In the case of $R=500$, the problem is solved for the six values of the forcing Strouhal number of $\pi / 6, \pi / 5, \pi / 4, \pi / 3,2 \pi / 5$ and $\pi / 2$. On the other hand, the case of $R=10^{3}$ is only investigated for $S=\pi / 4$ and $\pi / 2$. The peak rotation rate $\alpha_{m}$ for these cases is taken as 1.0. The results are presented below in the form of time variation of the streamline patterns as well as the variations of the drag and lift coefficients with time.

\subsection{Streamline patterns and force coefficients at $R=500$ and $\alpha_{m}=1.0$}

In figure 3 results for the case of $S=\pi / 6$ are shown for selected values of $t$ between $t=24$ and $t=36$. The chosen interval covers the third complete oscillation cycle following the start of the fluid motion. During the first half of the third cycle the rotational oscillation is in the counterclockwise sense. Close to the cylinder, figure 3(a), a clockwise vortex exists and is convected downstream with the aid of the counterclockwise rotation of the cylinder. This continues until figure $3(c)$ and a small counterclockwise circulation vortex in the upper right half of the cylinder is formed after figure 3(c). The reverse circulation region formed by the vortex and the flow from either side is negligible. When the clockwise rotation starts, the aforestated procedure is repeated in the opposite direction. The repetitive nature of the flow field in the near wake can be observed from mirror resemblance between the diagrams $3(a, d), 3(b, e), 3(c, f), 3(d, g)$. Figures $3(a)$ and $3(g)$ are almost the same, showing the periodic variation of the flow field at $t=24$ and $t=36$, respectively. Here we are dealing with a rotational oscillation frequency which is lower than the Kármán shedding frequency $\left(f / f_{0}=0.833\right)$ and the vortices in the near wake are simply the result of one vortex shedding in each half-cycle. They are shed at the Kármán shedding frequency; a clockwise vortex is formed on the lower half of the cylinder and a counterclockwise vortex is formed on the upper half. Thus non-synchronized vortex patterns are formed in the near wake.

The force coefficients are shown in figure 4 for $S=\pi / 6$ where $C_{D F}$ and $C_{L F}$ are the coefficients due to the friction and $C_{D P}$ and $C_{L P}$ are the coefficients due to the pressure. The contribution of frictional forces to the total drag and lift coefficients $C_{D}$ and $C_{L}$ is relatively small. From a numerical point of view, it may be noted that the oscillating amplitude should be large enough to avoid a long transition period and to facilitate periodic vortex formation; it typically takes only one cycle to reach a periodic pattern. This may be expected since the flow oscillations in this case exhibit low frequency and a relatively large amplitude. In addition, the $C_{D}$ curve oscillates twice as many times as the $C_{L}$ curve does after the initial transition period. This fact reflects the alternate vortex shedding in each cycle.

As the oscillating frequency increases to $S=\pi / 5$ and $S=\pi / 4$, synchronized vortex modes (not shown here due to the limitations of space) become locked 

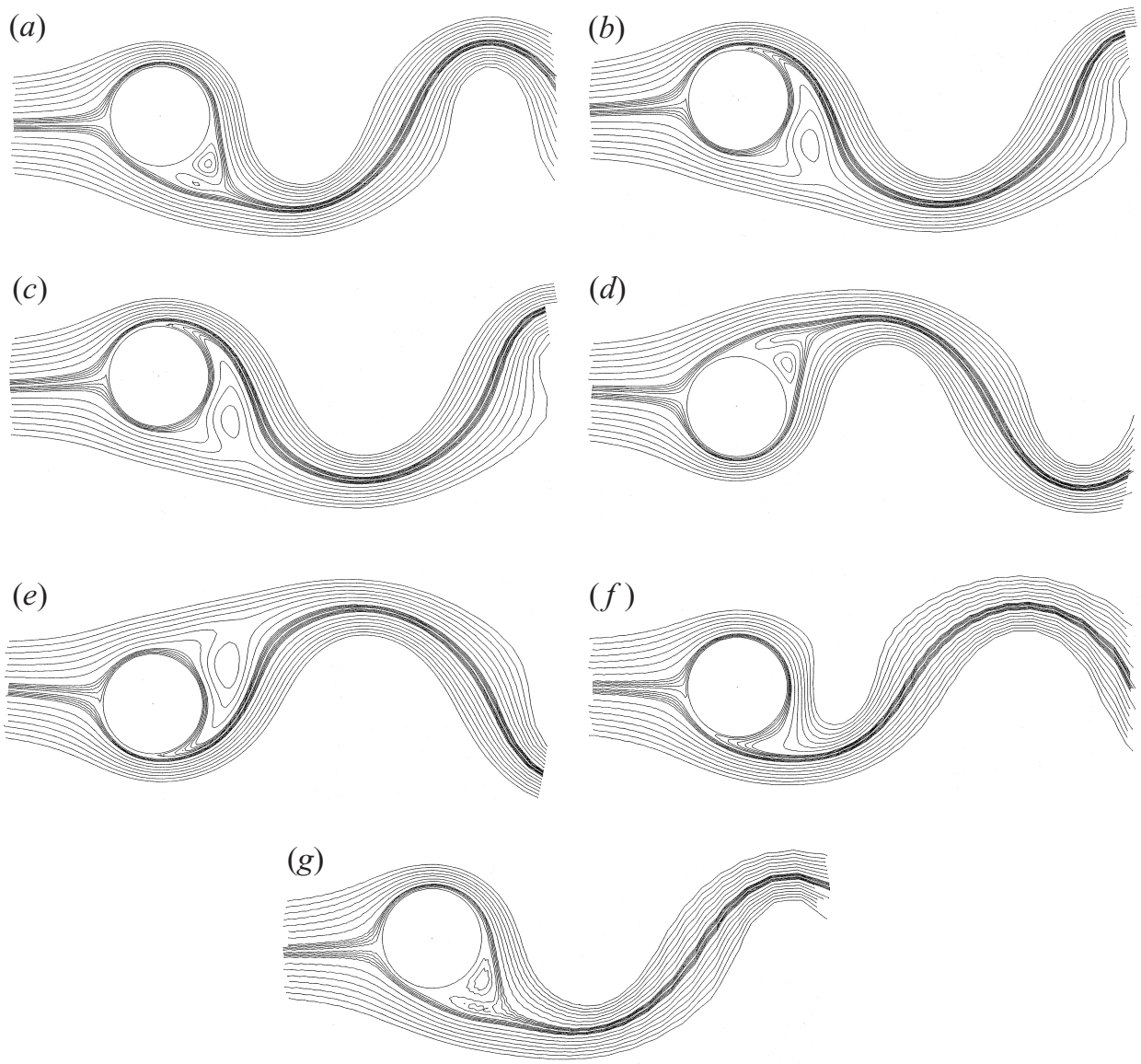

FIGURE 3. Instantaneous streamlines of the flow for $R=500: S=\pi / 6, \alpha_{m}=1.0:(a) t=24$, (b) $t=26,(c) t=28,(d) t=30,(e) t=32,(f) t=34,(g) t=36$.

to the cylinder oscillations. At $S=\pi / 5$ two opposite-sign vortices are shed from either side of the cylinder in each half-cycle. At $S=\pi / 4$, however, in the near wake, only one vortex is shed in a half-cycle. At a higher oscillation frequency, when $S=\pi / 3$, vortex shedding is also synchronized with the cylinder oscillation and the rotational oscillation frequency is higher than the Kármán vortex shedding frequency $\left(f / f_{0}=1.66\right)$. The instantaneous streamlines are displayed during the counterclockwise half-period in figure $5(a-d)$. As the cylinder starts to rotate in the counterclockwise direction, a reactive clockwise circulation is generated in the region $45^{\circ}<\theta<90^{\circ}$, figure $5(b)$. The accelerated flow during the last half-period and the flow due to the cylinder rotation confront each other in the region $0<\theta<90^{\circ}$. As the angular velocity increases, the circulation becomes larger and is convected downstream with the aid of accelerated flow at the lower half of the cylinder. This continues until the rotation is reversed. After this reversal (see figure 5e), the flow in the lower half of the cylinder is repeated by generating counterclockwise circulation in the region $270^{\circ}<\theta<360^{\circ}$. The vortex formation in this case is similar to the classical Kármán vortex street with a regular spacing. We note that as the forcing Strouhal number increases from $\pi / 5$ to $\pi / 3$ the horizontal and vertical spacings between vortices are observed to be inversely proportional to the forcing Strouhal number. 

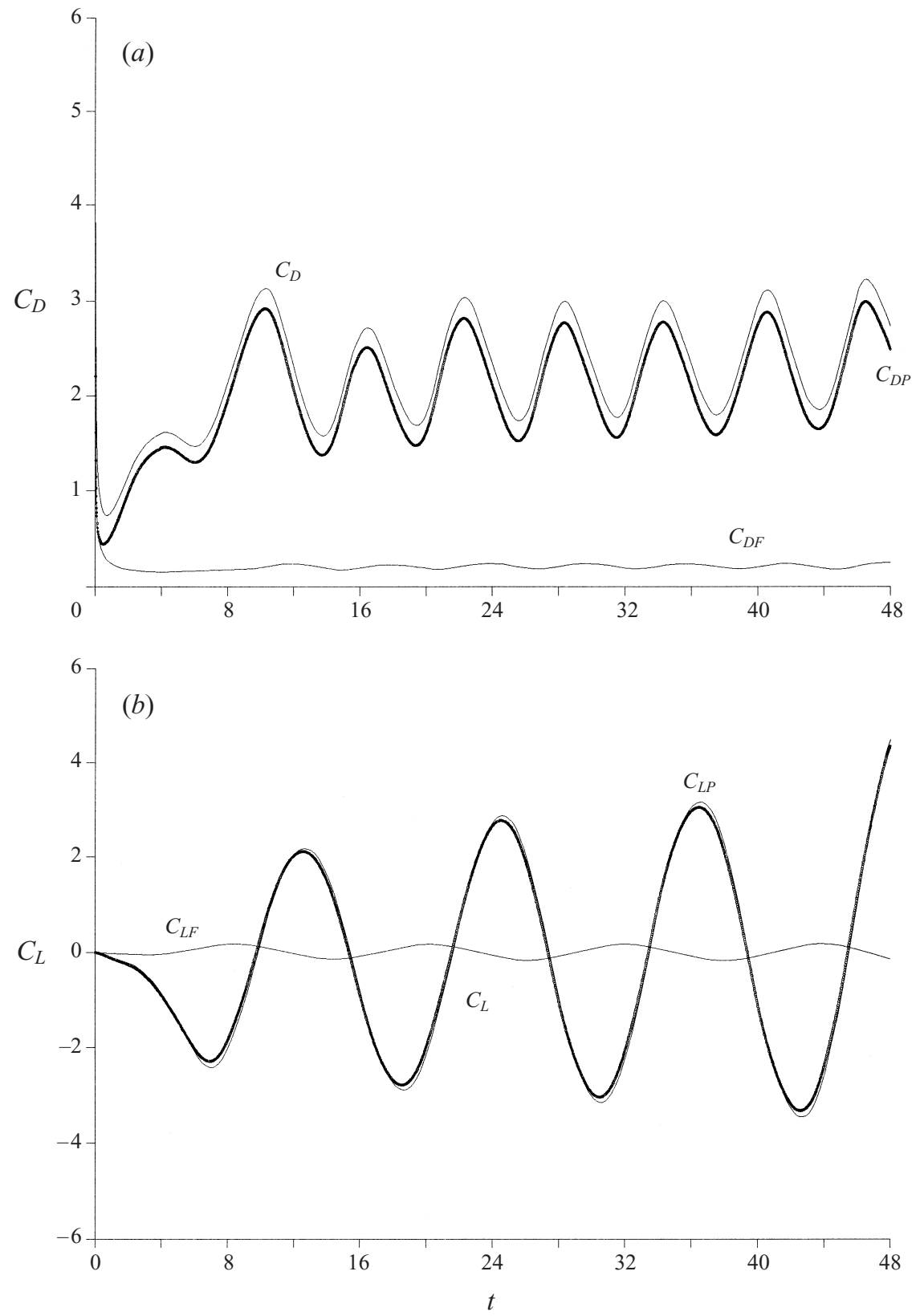

FigURE 4. Variation of $(a)$ the drag coefficients: $C_{D F}, C_{D P}$ and $C_{D}$ and $(b)$ the lift coefficients: $C_{L F}$, $C_{L P}$ and $C_{L}$, with $t$ at $R=500, S=\pi / 6$ and $\alpha_{m}=1.0$.

Figures 6-8 show the variations of the force coefficients at $S=\pi / 5, \pi / 4$ and $\pi / 3$. The lift coefficients vary at the cylinder oscillation frequency, and the drag coefficients oscillate at twice the cylinder oscillation frequency. The values of $C_{D}$ tend to be smaller as the forcing Strouhal number increases (or as amplitude becomes smaller) whereas $C_{L}$ increases. This is probably due to the fact that, when the cylinder begins to oscillate, it takes some time before boundary-layer separation occurs on the side 
The flow induced by a rotationally oscillating and translating circular cylinder 135

(a)

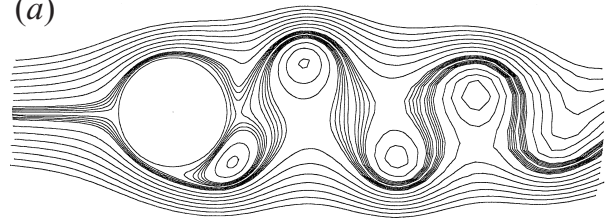

(c)

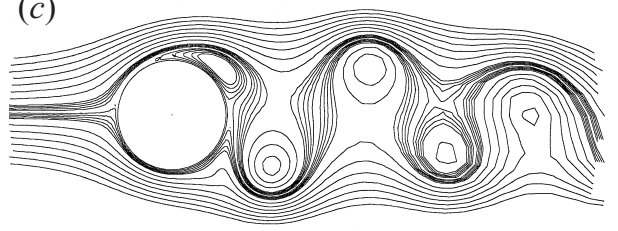

(e)

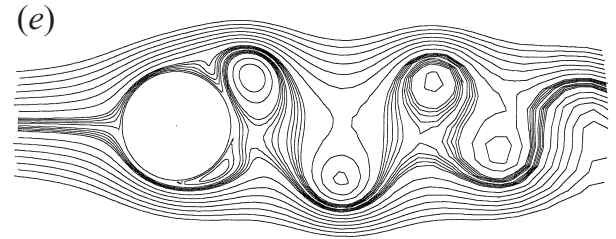

(b)

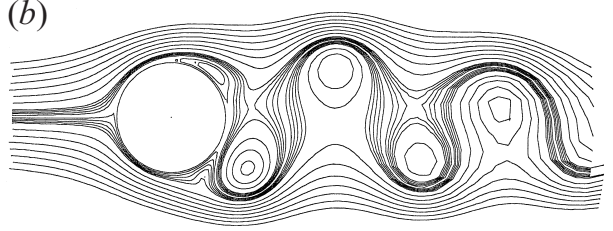

(d)

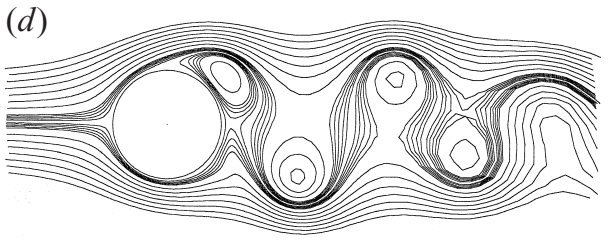

$(f)$

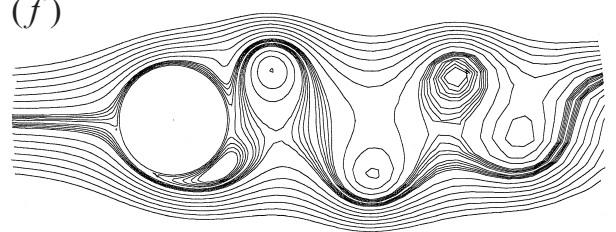

$(g)$

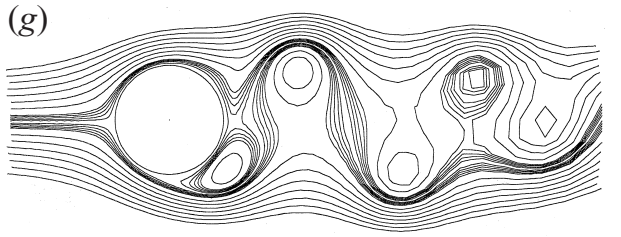

FiguRE 5. Instantaneous streamlines of the flow for $R=500: S=\pi / 3, \alpha_{m}=1.0$ : (a) $t=24$, (b) $t=26$, (c) $t=28,(d) t=30,(e) t=32,(f) t=34,(g) t=36$.

of the cylinder astern of the oscillation direction. Therefore, in the small-amplitude and high-frequency case, the region of the next cycle of oscillation is entered before enough separation takes place. This reduces the drag forces.

In the near wake, the vortex patterns at $S=2 \pi / 5$ (not shown here) and $S=\pi / 2$ (shown in figure 9) are also synchronized with the cylinder oscillation and the vortex modes are similar to those for lower frequencies $(S=\pi / 6, \pi / 5)$, showing markedly periodic behaviour. In the case of $S=\pi / 2$, the computations were carried out over eight complete cycles. During the first two cycles when $0<t<8$ (not shown here), the usual formations and detachments of upper (clockwise) and lower (counterclockwise) vortex pairs take place. Figure $9(a-e)$ shows five snapshots of the flow covering the third complete cycle. In this cycle the near-wake structure is quite different from the ones obtained in the previous two cycles. During the interval $9 \leqslant t \leqslant 10$ the clockwise vortex pair which was shed in the second cycle joins with the clockwise vortex pair formed in the third cycle: the vortices of the same sign coalescence. With the increase of time this double vortex pair becomes weak and moves downstream (see figure $9 e, f$ ) and fades away at $t=13.0$ (figure $9 g$ ). In the time interval $13<t<24$ no co-rotating vortex pair appears but in the seventh cycle (figure $9 h-l$ ) we once again observe shedding of a double counterclockwise vortex pair from the lower half of the cylinder and the movement of it in the downstream direction. This co-rotating vortex pair fades away at the end of the seventh cycle and a new double clockwise 


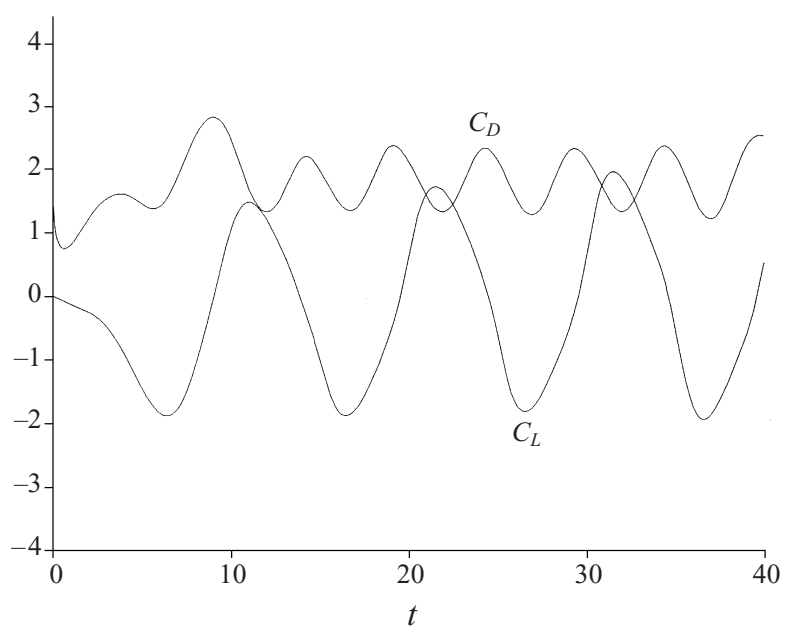

FIGURE 6. Variation of the drag and lift coefficients, $C_{D}$ and $C_{L}$ with $t$ at $R=500$, $S=\pi / 5$ and $\alpha_{m}=1.0$.

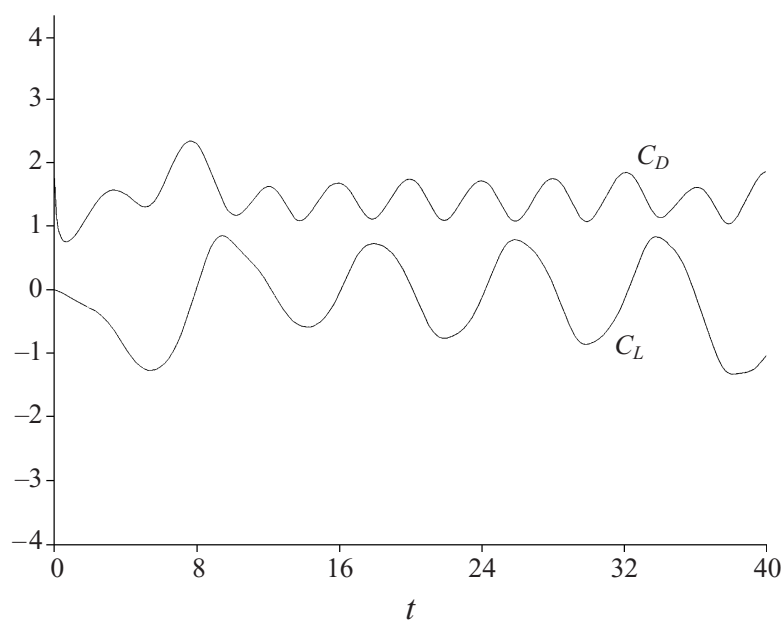

FigURE 7. As figure 6 but for $S=\pi / 4$.

vortex pair appears at the beginning of the eighth cycle (see figure $9 \mathrm{~m}$ ). It seems that this phenomenon will be repeated with vortex pairs that are shed from the upper and lower half of the cylinder, when the flow has settled down to a truly periodic state.

It may be noted that this unusual feature in the wake, the presence of two corotating vortices, was first reported by $\mathrm{Lu} \&$ Sato (1996) in the same type of flow (see figure 16, p. 844). In their work this phenomenon was observed in a different parameter range from ours: $\left(R=10^{3}: f / f_{0}=3.0\right.$ and $\left.\alpha=3.0\right)$ by investigating the equi-vorticity lines during only one cycle of oscillation. Thus, unlike the present work, a periodic repetition of the phenomenon was not observed. The fact that there is a periodic motion of this kind is one of the essentially new contributions of our work.

This kind of vortex pair interaction in the near wake might be due to the rotary motion of the cylinder which tends to attract the two vortex pairs shedding away from the cylinder to each other, thus slowing down their convection further downstream. 
The flow induced by a rotationally oscillating and translating circular cylinder 137

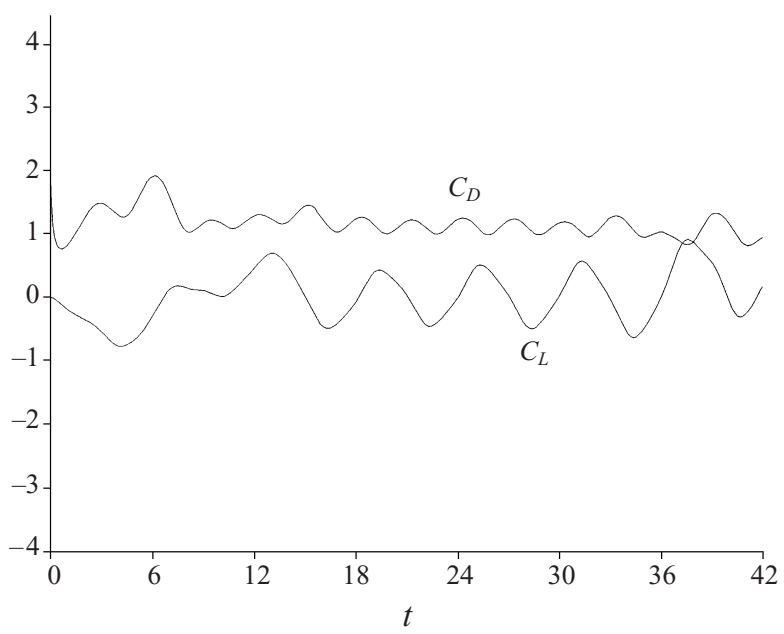

Figure 8. As figure 6 but for $S=\pi / 3$.

By inspecting the lower forcing frequency cases we see that the near-wake region is much more periodic and organized.

The calculated values of $C_{D}$ and $C_{L}$ in the case of $S=\pi / 2$ are plotted in figure 10 . The lift coefficient oscillates at the same frequency as the cylinder oscillation as expected. At this forcing Strouhal number we observe an interesting behaviour of the drag coefficient: the $C_{D}$ curve oscillates at twice the frequency of cylinder oscillation until $t=32$; beyond this time it oscillates at the same frequency as the cylinder oscillation. This behaviour might be due to the coalescence of vortices of the same sign.

\subsection{Streamline patterns and force coefficients at $R=10^{3}$ and $\alpha_{m}=1.0$}

To discuss the effect of the Reynolds number on the vortex patterns and force coefficients, the flow is calculated at $S=\pi / 4$ and $\pi / 2$. At $S=\pi / 4$ the vortices in the near wake (not shown here) are simply the result of one vortex shedding each halfcycle. This is the classical mode of single-vortex-shedding in each half-cycle leading to the formation of the Kármán street and the vortices in the near wake are shed at the rate of oscillation experienced by the cylinder. Thus for the value of the forcing frequency $S=\pi / 4$, we find that the main features of the near wake are nearly the same for $R=500$ and $R=10^{3}$.

In the case of $S=\pi / 2$, the computations are carried out over four complete cycles. Figure 11 illustrates the flow for this case at four instants in time during the fourth cycle of oscillation. Comparison of these figures with the corresponding ones in the case of $R=500$ indicates that the near wake does not involve adjacent co-rotating vortex coalescence in the case of $R=10^{3}$ unlike the case of $R=500$. Thus the Reynolds number seems to have more influence on the flow structures at higher values of $S$ than at lower values of $S$.

The calculated values of $C_{D}$ and $C_{L}$ are plotted in figures 12 and 13 for the cases of $S=\pi / 4$ and $S=\pi / 2$. In each case the contribution of frictional forces to the drag $C_{D}$ and lift $C_{L}$ coefficients is relatively small and the main contribution comes from the pressure forces since in this type of flow at moderate and high Reynolds numbers the viscous flow effect is limited to the thin boundary layer and the subsequent narrow near-wake region. It is noted that in figure $13(b)$ the lift coefficient $C_{L}$ becomes 
(a)

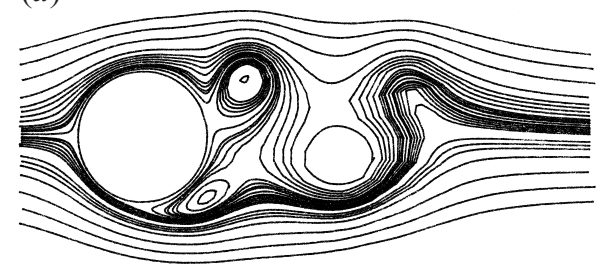

(c)

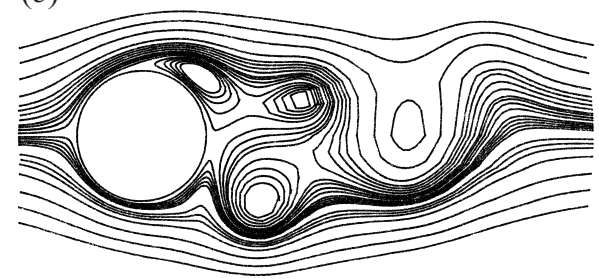

(b)

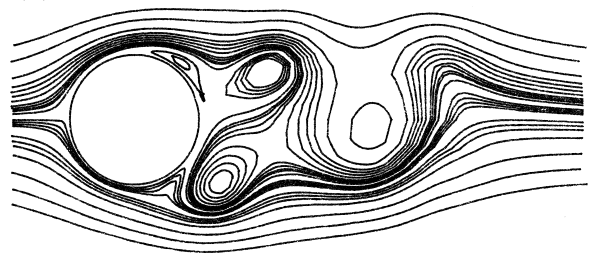

(d)

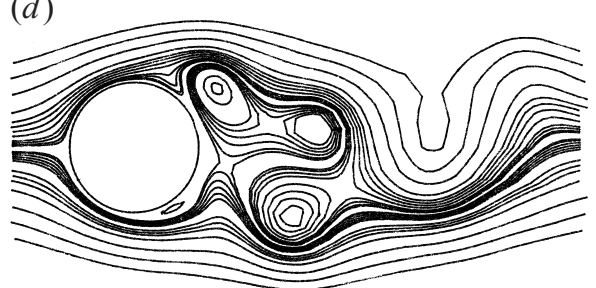

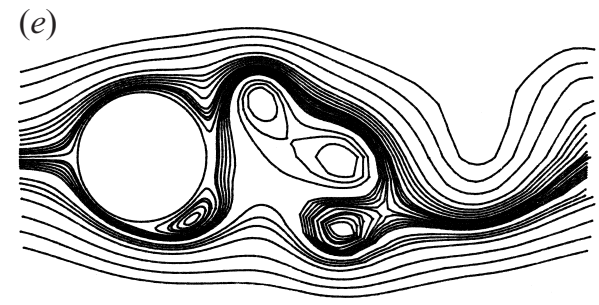

FIGURE 9. For caption see facing page.

periodic for $t>12$ but the time-averaged lift is non-zero; there appears to be a 'preferred direction' for the lift which is in some way associated with the vortex shedding from the cylinder, i.e. there will be a loss of lift as vortices are shed from the cylinder. Thus there may be a lack of balance in the situation as the cylinder rotates one way or the other depending on how the vortices are shed, which in turn presumably depends on the asymmetry of the initial motion.

\section{Conclusions}

An implicit time-marching scheme was utilized to analyse the vortex formation modes behind a cylinder as well as the fluid forces acting on a cylinder, where the uniformly translating cylinder was in time-dependent rotational oscillation. The numerical scheme was verified by applying it to study the special case of a steadily rotating and translating circular cylinder. Furthermore, the simulations of the startup flow at a low Reynolds number are consistent with Taneda's (1978) experimental results.

Calculations are performed in a different parameter range from those used in previous numerical studies. Two different Reynolds numbers are considered. In the case of $R=500$ the results are obtained in a range of forcing Strouhal number $\pi / 6 \leqslant S \leqslant \pi / 2$ or oscillating frequencies $0.833 \leqslant f / f_{0} \leqslant 2.5$ when the peak-rotational rate is $\alpha=1.0$. An interesting phenomenon occurs for sufficiently large time when $R=500$ and $S=\pi / 2$ : the two co-rotating vortex pairs are shed away from the cylinder to form a double co-rotating vortex pair which slows down their convection 
The flow induced by a rotationally oscillating and translating circular cylinder 139
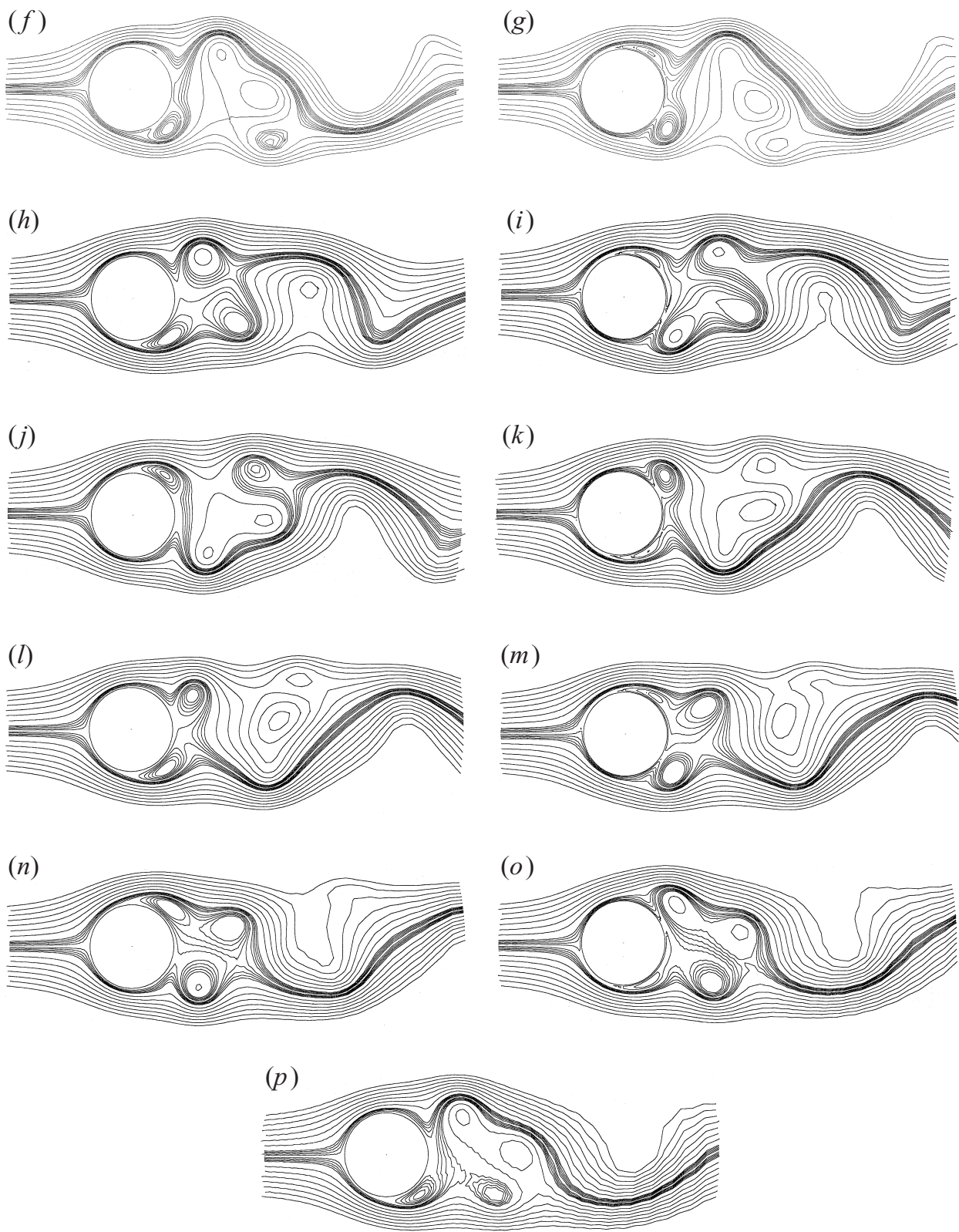

FigURE 9. Instantaneous streamlines of the flow for $R=500: S=\pi / 2, \alpha_{m}=1.0:(a) t=8,(b) t=9$, (c) $t=10,(d) t=11,(e) t=12,(f) t=12.5,(g) t=13,(h) t=24,(i) t=25,(j) t=26,(k) t=27$, (l) $t=28,(m) t=29,(n) t=30,(o) t=31,(p) t=32$.

further downstream. This seems to delay the development of the periodic flow pattern in the near wake.

When $f$ is lower than the natural shedding frequency, an initial clockwise vortex is formed on the lower half of the cylinder when the cylinder is rotated in the counterclockwise direction and a counterclockwise vortex is formed on the upper half when the clockwise rotation starts. This leads to a non-synchronized vortex formation mode and as a result of this the lift and drag are affected by the frequencies $f_{0}$ and 


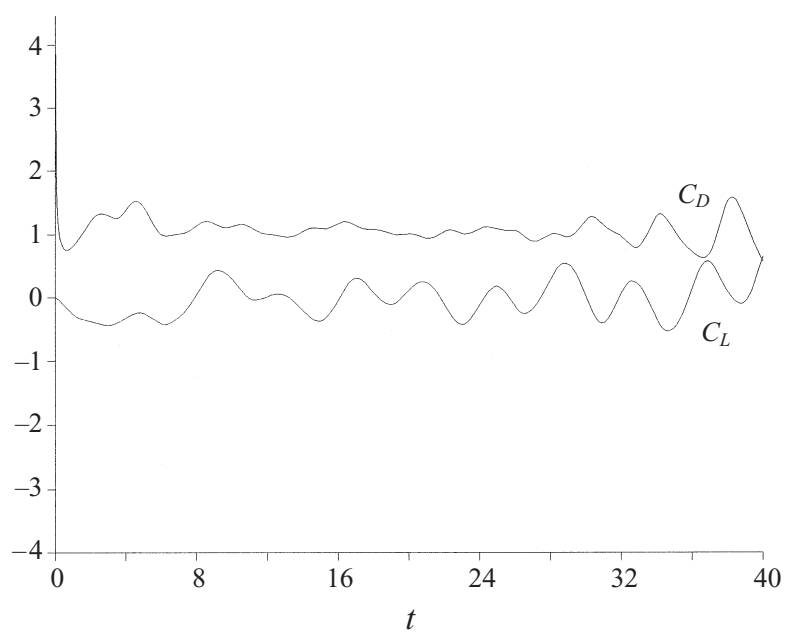

FIGURE 10. Variation of the drag and lift coefficients, $C_{D}$ and $C_{L}$ with $t$ at $R=500$, $S=\pi / 2$ and $\alpha_{m}=1.0$.

(a)

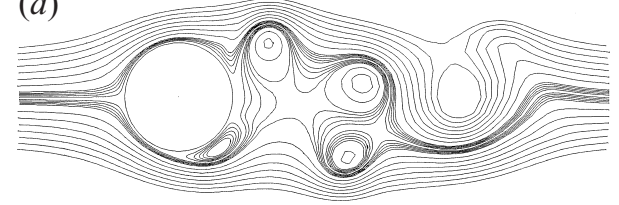

(c)

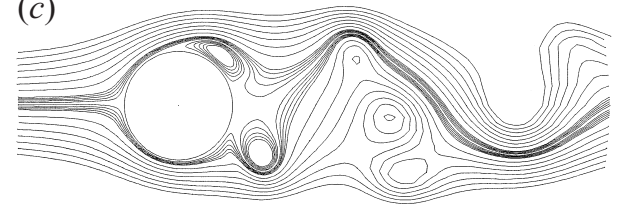

(b)

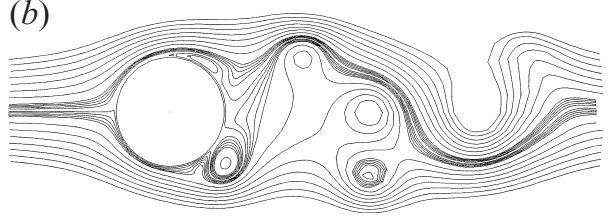

(d)

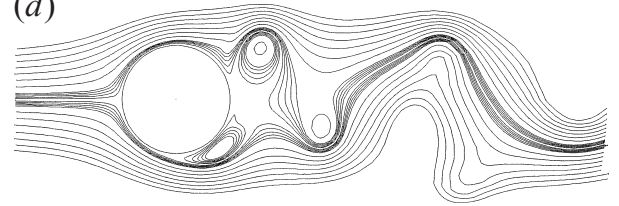

FIGURE 11. Instantaneous streamlines of the flow for $R=10^{3}: S=\pi / 2, \alpha_{m}=1.0:(a) t=12$, (b) $t=13,(c) t=14,(d) t=16$.

$2 f_{0}$, respectively. A salient vortex formation mode change was observed when the forcing oscillation frequency $f$ approximates the natural shedding frequency, namely a synchronized vortex mode is found to be locked to the cylinder oscillations. When $f$ is higher than the Kármán vortex-shedding frequency $\left(f_{0}=0.1\right)$ an initial reactive clockwise vortex is formed on the upper half of the cylinder when the cylinder is rotated in the counterclockwise direction and a counterclockwise vortex is formed on the lower half when the clockwise rotation starts. This leads to a synchronized vortex mode as the vortices in the near wake are shed at the same frequency as the cylinder oscillation.

In addition, the lift coefficients vary at the cylinder oscillation frequency, and the drag coefficients oscillate at twice the cylinder oscillation frequency. In the case of $S=\pi / 2$ or $f / f_{0}=2.5$ we observe an interesting behaviour of the drag coefficient: the $C_{D}$ curve oscillates at twice the frequency of the cylinder oscillation until $t=32$; beyond this time it oscillates at the same frequency as that of the cylinder oscillation. We believe that this behaviour is due to coalescence of the vortices of the same sign observed in the flow patterns when $S=\pi / 2$. This kind of behaviour in the drag 
The flow induced by a rotationally oscillating and translating circular cylinder 141
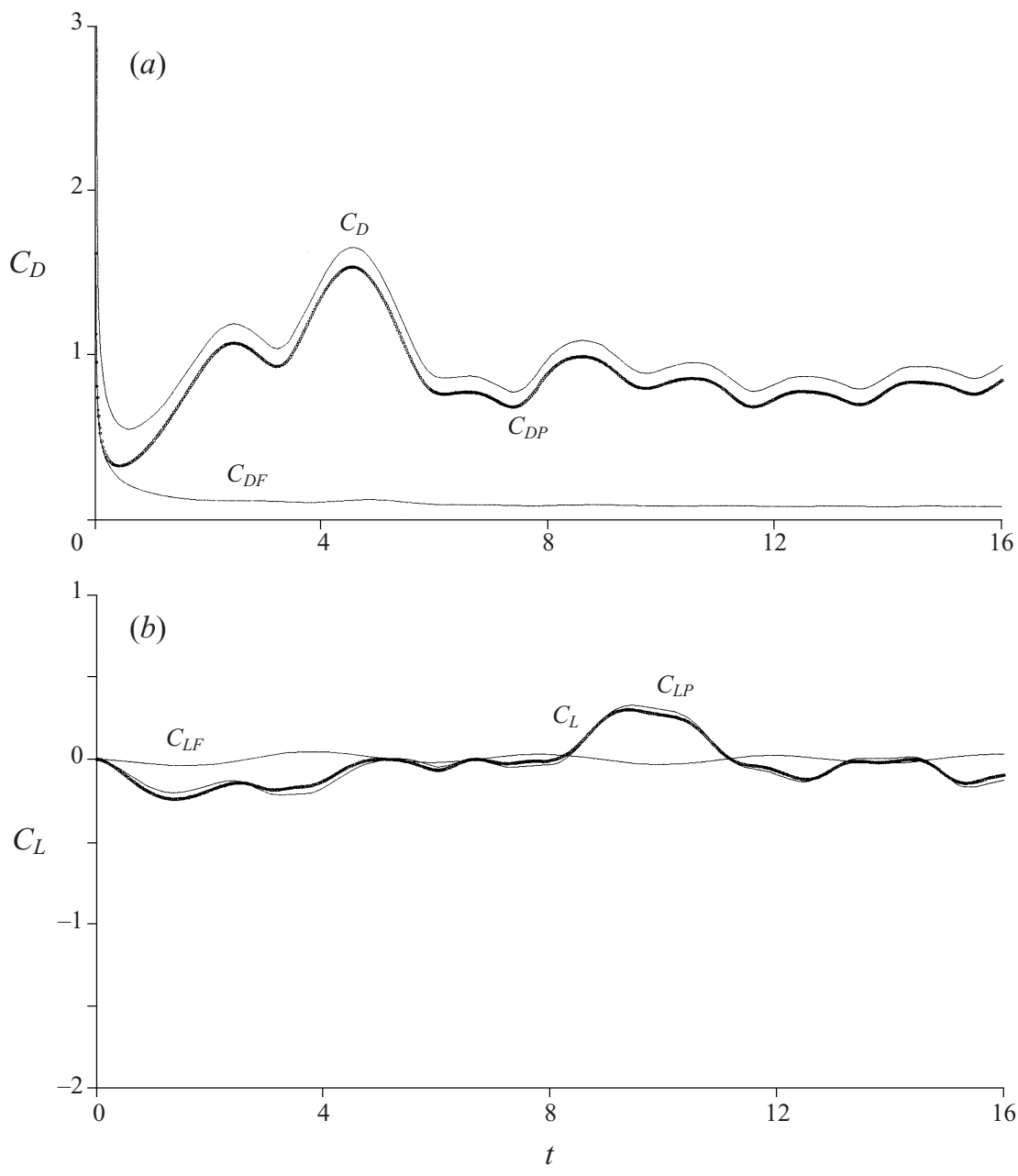

FIGURE 12. Variation of $(a)$ the drag coefficients, $C_{D}$ and $(b)$ the lift coefficients, $C_{L}$, with $t$ at $R=10^{3}, S=\pi / 4$

coefficient in this type of flow has not been observed previously and seems to make a quite new contribution to the fluid mechanics of the problem. It is also observed that as the forcing oscillation frequency increases from $S=\pi / 6$ to $S=\pi / 2$ the value of the drag coefficient decreases by about $50 \%$. Drag reduction was also obtained by Tokumaru \& Dimotakis (1991), among other phenomena, when both the forcing frequency and the peak-rotational rate are somehow higher than usual.

In the case of $R=10^{3}$ results are obtained at $S=\pi / 4$ and $\pi / 2$ or $f / f_{0}=1.25$ and 2.5 when the peak-rotational rate is $\alpha=1.0$. For $S=\pi / 4$, the near-wake structure is nearly the same as in the case of $R=500$. It is found that for $S=\pi / 2$ the near wake does not involve adjacent co-rotating vortex coalescence and as a result of this the usual behaviour of the drag coefficient is observed unlike when $R=500$. Thus the Reynolds number seems to have more influence on the structures and the fluid forces at higher values of $S$ than at lower values, which is in contrast with the findings of $\mathrm{Lu} \&$ Sato (1996). In their work it is reported that the large-scale vortex structures in the near wake remain nearly the same for $R=200,10^{3}$ and $3 \times 10^{3}$. 

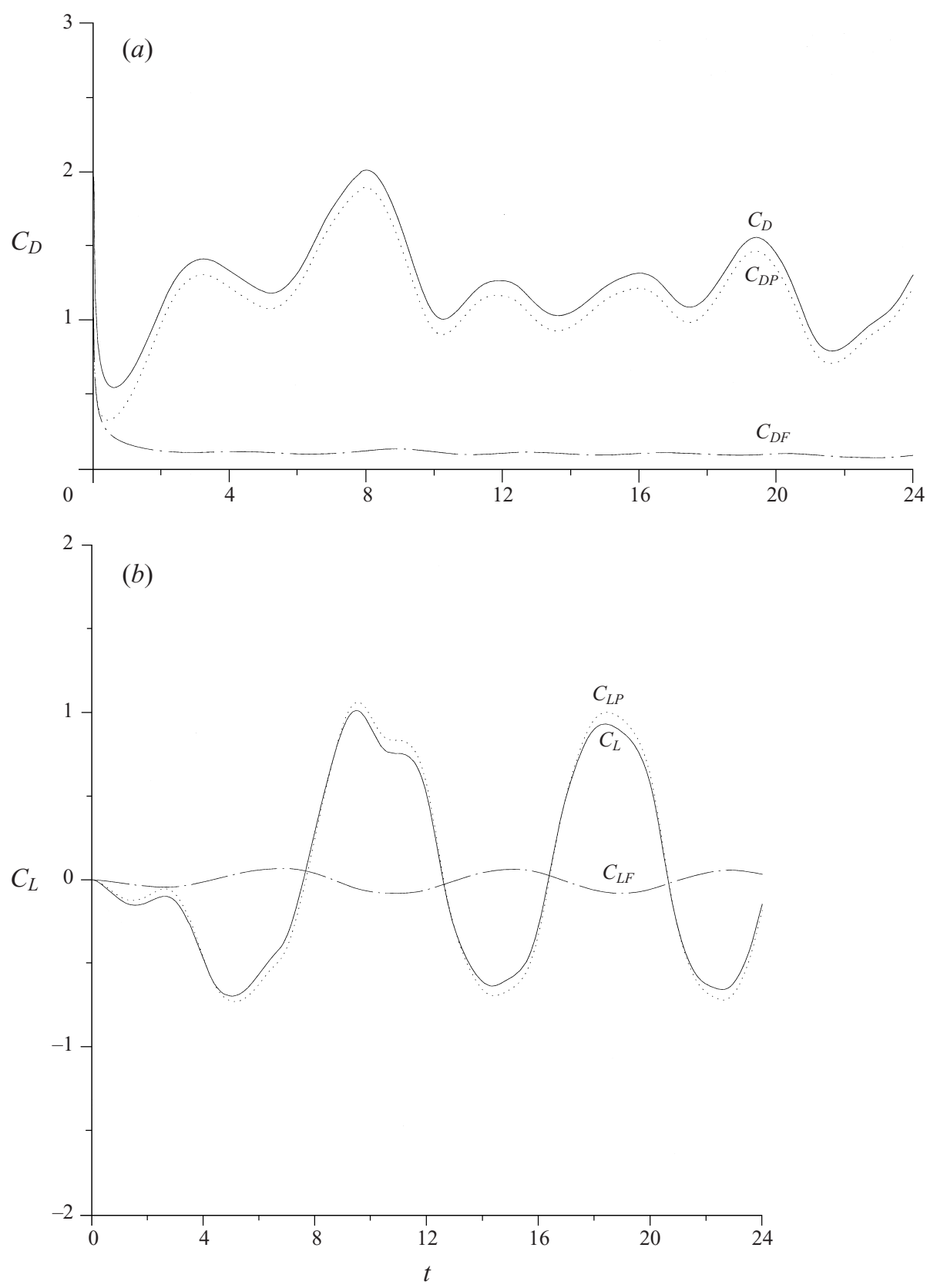

Figure 13 . As figure 12 but for $S=\pi / 2$.

In summary, therefore, we have observed some new fluid mechanical phenomena in this type of flow, particularly with regard to the periodicity of the double co-rotating vortex shedding and the behaviour of the drag coefficient for certain values of $R$ and $S$. We have also confirmed instances of drag reduction with increasing Strouhal number found by others but have found more influence of the Reynolds number of the fluid structures than that found in previous work. 
The flow induced by a rotationally oscillating and translating circular cylinder 143

We would like to acknowledge that our recent studies on oscillatory flows owe much to Professor Norman Riley and we thank him for several useful comments during the course of this research. The support of the Natural Sciences and Engineering Council of Canada for this investigation is also gratefully acknowledged.

\section{REFERENCES}

Badr, H. M., Coutanceau, M., Dennis, S. C. R. \& Menard, C. 1986 On the phenomenon of vortex transposition of coalescence in separated flows. C. R. Acad. Sci. Paris 302(II), 1127-1130.

Badr, H. M., Coutanceau, M., Dennis, S. C. R. \& Menard, C. 1990 Unsteady flow past a rotating circular cylinder at Reynolds numbers $10^{3}$ and $10^{4}$. J. Fluid Mech. 220, 459-484.

BADR, H. M. \& Dennis, S. C. R. 1985 Time-dependent viscous flow past an impulsively started rotating and translating circular cylinder. J. Fluid Mech. 158, 447-488.

Badr, H. M., Dennis, S. C. R. \& KocabiYIK, S. 1995a The initial oscillatory flow past a circular cylinder. J. Engng Maths 29, 255-269.

BadR, H. M., Dennis, S. C. R. \& KocabiYiK, S. 1996 Symmetrical flow past an accelerated circular cylinder. J. Fluid Mech. 308, 97-110.

Badr, H. M., Dennis, S. C. R., KocabiYiK, S. \& NguYen, P. 1995b Viscous oscillatory flow about a circular cylinder at small to moderate Strouhal number. J. Fluid Mech. 303, 215-232.

BAEK, S.-J. \& SUNG H. J. 1998 Numerical simulations of the flow behind a rotary oscillating circular cylinder Phys. Fluids 10, 869-876.

Bearman, P. W. 1984 Vortex shedding from oscillating bluff bodies. Ann. Rev. Fluid Mech. 16, $195-222$.

Berger, E. \& Willie, R. 1972 Periodic flow phenomena. Ann. Rev. Fluid Mech. 4, 313-340.

Chang, C.-C. \& Chern, R.-L. 1991 Vortex shedding from an impulsively started rotating and translating circular cylinder. J. Fluid Mech. 233, 265-298.

Chen, Y.-M., Ou, Y.-R. \& Pearlstein A. J. 1993 Development of the wake behind a circular cylinder impulsively started into rotatory and rectilinear motion. J. Fluid Mech. 253, 449-484.

Chou, M.-H. 1997 Synchronization of vortex shedding from a cylinder under rotary oscillation Computers Fluids 26, 755-774.

Coluns, W. M. \& Dennis, S. C. R. 1973 Flow past an impulsively started circular cylinder. J. Fluid Mech. 60, 105-127.

Coutanceau, M. \& Menard, C. 1985 Influence of rotation on the near-wake development behind an impulsively started circular cylinder. J. Fluid Mech. 158, 399-446.

D’Alessio, S. J. D., Dennis, S. C. R. \& NGuYen, P. 1999 Unsteady viscous flow past an impulsively started oscillating and translating elliptic cylinder. J. Engng Maths 35, 339-357.

Daube, O. 1992 Resolution of the 2D Navier-Stokes equations in velocity-vorticity formulation by means of an inluence matrix technique. J. Comput. Phys. 103, 402-414.

DenNis, S. C. R. \& KoCABIYIK, S. 1991 An asymptotic matching condition for unsteady boundarylayer flows governed by the Navier-Stokes equations. IMA J. Appl. Maths 47, 81-98.

Dennis, S. C. R. \& QuartapelLE, L. 1989 Some uses of Green's Theorem in solving the NavierStokes equations. Intl J. Numer. Meth. Fluids 9, 871-890.

Filler, J. R., Marston, P. L. \& Min, W. C. 1991 Response of the shear layers separating from a circular cylinder to small amplitude rotational oscillations. J. Fluid Mech. 231, 481-499.

Griffin, O. M. \& HaLL, M. S. 1991 Review-Vortex shedding lock-on and flow control in bluff body wakes. Trans. ASME: J. Fluids Engng 113, 526-537.

Kiм, J. \& MorN, P. 1985 Application of a fractional step method to incompressible Navier-Stokes equations. J. Comput. Phys. 59, 308-323.

KOCABIYIK, S. $1996 a$ Boundary layer on a sphere in an oscillating viscous flow. Can. Appl. Math Q. 4, 97-107.

KocABIYIK, S. 1996b On an accelerated flow around a sphere. Appl. Maths Lett. 9, 41-46.

KocAbIYIK, S. \& NGUYEN, P. 1996 A finite difference calculation for transverse superimposed oscillation. Can. Appl. Maths Q. 9, 381-419.

Lu, X.-Y. \& SATo, J. 1996 A numerical Study of flow past a rotationally oscillating circular cylinder J. Fluids Struct. 10, 829-849. 
Nair, M. T., Sengupta, T. K. \& Chauhan U. S. 1998 Flow past rotating cylinders at high Reynolds numbers using higher order upwind scheme. Computers Fluids 27, 47-70.

Okajima, A., TAKata, H. \& Asanuma T. 1981 Viscous flow around a rotationally oscillating circular cylinder. Ins. Space and Aero. Sci. (U. Tokyo), Rep. 532.

Riley, N. \& Wybrow, M. F. 1995 The flow induced by the torsional oscillations of an elliptic cylinder. J. Fluid Mech. 290, 279-298.

StEgGel, N. \& RocklifF, N. 1997 Simulations of the effects of body shape on lock-in characteristics in pulsating flows by the discrete vortex method. J. Wind Engng Indst. Aero. 69-71, 317-329.

SüMer, B. M. \& FredsøE, J. 1997 Hydrodynamics around Cylindrical Structures. World Scientific.

TANEDA, S. 1978 Visual observations of the flow past a circular cylinder perfoming a rotatory oscillation. J. Phys. Soc. Japan 45, 1038-1043.

Tokumaru, P. T. \& Dimotakis, P. E. 1991 Rotary oscillation control of a cylinder wake. J. Fluid Mech. 224, 77-90.

Williamson, C. H. K. 1988 The existence of two stages in the transition to three-dimensionality of a cylinder wake. Phys. Fluids 31, 3165-3168.

Wu, J., Mo, J. \& VAKILI, A. 1989 On the wake of a cylinder with rotational oscillations. AIAA Paper 89-1024. 